\title{
Anticipatory behaviour deficits in closed head injury
}

\author{
P E FREEDMAN,* JOSEPH BLEIBERG, $\dagger$ KENNETH FREEDLAND $\dagger$ \\ From the University of Illinois at Chicago, ${ }^{*}$ and Northwestern University Medical School, $\dagger$ Chicago, USA
}

SUMMARY Closed head injured patients demonstrated greater anticipatory behaviour deficit than cerebral vascular accident patients on a shuttlebox-analog avoidance task, even though these two groups did not differ on escape behaviour and on performance on the individual tests of the Halstead-Reitan Battery and Wechsler scales. Neither clarification of instructions, additional trials, nor enhancement of the warning cue appeared to improve the anticipatory behaviour deficit. It was concluded that anticipatory behaviour deficit should be considered a relatively dense deficit that is not identified by standard instruments. Clinical and practical implications are discussed.

Anticipatory behaviour deficits in humans following brain injury long have been described in the clinical and theoretical literature and recently have been studied empirically. Although the term anticipatory behaviour deficit was not used, Luria ${ }^{12}$ described patients with frontal lobe lesions as showing deficits in planning and regulating behaviour in anticipation of expected consequences. Denny-Brown ${ }^{3}$ noted "inability to visualise consequences" as a central deficit following leucotomy. Others, such as Oppenheimer ${ }^{4}$ and Nauta $^{5}$ have reported similar observations, with Nauta directly referring to an impairment of the "anticipatory selection process."

Homologous deficits have been found in prefrontally lesioned primates. Ablation of the prefrontal cortex, especially the orbital and medial portions, has been shown to interfere with avoidance learning in primates. ${ }^{6-8}$ These findings are not attributable to reduced responsivity to aversive stimulation per se, since prefrontal primates become agitated in the presence of an aversive warning stimulus and attempt to escape from an aversive noxious stimulus. $^{910}$ Thus, although these subjects are able to escape from aversive stimulation, they are unable to avoid it.

A recent study quantified anticipatory behaviour deficit and compared its prevalence in patients with middle or posterior cerebral artery cerebrovascular accident (CVA) and patients with closed head injury

Address for reprint requests: Dr PE Freedman, Department of Psychology, University of Illinois at Chicago, Box 4348, Chicago, Illinois 60680 .

Received 8 November 1985 and in revised form 14 January 1986. Accepted 24 February 1986
(CHI). Bleiburg, Freedman, Schueneman, Merbitz and Swartz $\mathrm{z}^{11}$ operationalised anticipatory behaviour deficit using an escape-avoidance task, with failure to learn the avoidance response considered a reflection of anticipatory behaviour deficit. The brain damaged group, as a whole, showed impairment of avoidance but not of escape learning when compared to normap and non-neurological control subjects. When the brain damaged group was separated into CVA and $\mathrm{CHI}$ groups, the $\mathrm{CHI}$ group showed an avoidance deficit relative to the CVA group, even though the groups had equivalent degrees of cognitive impairment as measured by Halstead Impairment Indexes and showed no difference in escape learning.

The present study uses an entirely new sample of subjects to address several methodological issues raised by the Bleiberg, et $a l^{11}$ study and to assess the replicability of the original findings regarding disproportionate anticipatory behaviour deficit in $\mathrm{CHI}$ as compared to CVA patients.

Observation and post-experiment debriefing of experimental and control subjects in the original study suggested that the instructions to subjects were more ambiguous than perhaps was necessary. Ambiguous instructions are desirable in the anticipatory behaviour deficit paradigm because the intent is to assess the subject's ability to identify the potential for avoidance responses, and instructions that are too explicit with respect to requesting an avoidance response would defeat the intent of the procedure. The present study tested a more explicit set of instructions, the goal being to make the instructions as explicit as possible short of instructing the subject what to do. Explicit instructions have the advantage of reducing potential error variance in the results.

Another modification of procedure was made, also 
with the intent of reducing error variance. The original study required all subjects to complete 48 trials (40 avoidance opportunities and eight interspersed escape-only trials). Subjects who rapidly learned to avoid sometimes became bored with the procedure and intentionally made errors to break the monotony. To correct this, and to shorten the time required for the procedure, a criterion of eight out of ten successful avoidances was established, with testing terminated when the criterion was achieved.

To examine the possibility that subjects who did not acquire the avoidance response within 48 trials might do so if given more opportunity, subjects in the present study were given up to 96 trials to reach criterion. Moreover, to explore the hypothesis that the saliency of the warning stimulus (conditioned stimulus, CS) might be a factor in anticipatory behaviour deficit, a random half of the subjects who failed to learn in the first $\mathbf{4 8}$ trials had the saliency of the CS increased.

Shuttlebox avoidance conditioning was again used as the paradigm to measure anticipatory behaviour. This paradigm measures the acquisition of the active avoidance response (going from one side to the other when the signal (CS) comes on but before the noxious stimulus (unconditioned stimulus, UCS) onset). In addition, it allows for two more response measures. By punishing intertrial responding (spontaneous crossovers) with UCS presentation, a discriminant passive avoidance component ${ }^{12}$ is measured. And by introducing pre-programmed trials on which the CS and the UCS come on simultaneously, escape-only behaviour is monitored.

Therefore, using this paradigm, two types of anticipatory behaviour can be measured: active avoidance (excitatory) of the CS, and passive avoidance (inhibitory) of the cue of no CS. Also, nonanticipatory (demand) behaviour can be measured by looking at escape latencies on escape-only trials.

\section{Method}

\section{Subjects}

Experimental subjects consisted of patients referred for evaluation to the Neuropsychology Service of the Rehabilitation Institute of Chicago who met the following criteria: inclusion criteria were that the patient had a documented brain injury or lesion and had the ability to complete the HalsteadReitan Neuropsychological Test Battery. The exclusion criterion was a history of previous psychiatric illness or alcoholism. In the $\mathrm{CHI}$ group there were 15 males and six females with a mean age of $33.3(\mathrm{SD}=14 \cdot 7)$, mean education of $12 \cdot 16$ years $(\mathrm{SD}=3 \cdot 33)$, and mean chronicity of 14.8 months $(S D=30 \cdot 4)$. In the CVA group there were five males and two females with a mean age of $40.7(\mathrm{SD}=9 \cdot 0)$, mean education of $14 \cdot 1$ years $(\mathrm{SD}=2 \cdot 7)$, and mean chronicity of 8.6 months (SD $=11.0$ ).

Although the CVA group is on average too young to con- stitute a representative sample of the entire stroke population, it is representative of the stroke patients referred for neuropsychological testing in this setting. A relatively large proportion of the older stroke inpatients at this facility are either unable to tolerate testing or are not referred because testing has been deemed unnecessary.

Subjects then were categorised by lesion type using data obtained from their inpatient medical records (CT scans, skull radiographs, EEC's, and neurological examinations and histories). This resulted in three subgroups: (1) Closed Head Injury (CHI), consisting of subjects with closed head injury but no additional injuries such as depressed skull fracture or haematoma requiring surgical intervention $(\mathrm{N}=21)$; (2) Cerebral Vascular Accident (CVA), consisting of patients with a single CVA restricted to the middle or posterior cerebral artery distribution of either hemisphere $(\mathrm{N}=7)$; and (3) Other (OTH), consisting of patients with multiple CVAs, anoxia, multiple sclerosis, subarachnoid haemorrhage, and anterior communicating artery aneurysm $(N=20)$.

\section{Apparatus}

A $30 \mathrm{~cm}$ wide by $15 \mathrm{~cm}$ deep by $1.2 \mathrm{~cm}$ thick horizontal board formed the base of the human "shuttlebox." Affixed to its rear was a vertical board of the same dimensions used to hold two $24 \mathrm{v}$ yellow jeweled lamps which were mounted $3 \mathrm{~cm}$ from the top and either side.

The "shuttlebox" apparatus itself was a Plexiglas bar $29 \mathrm{~cm}$ long by $0.7 \mathrm{~cm}$ wide pivoted at its centre by a bolt to a steel bracket, allowing a maximum seesaw movement of $3.5 \mathrm{~cm}$ at the extremes. The bar was balanced by the levers of two microswitches on either side of the fulcrum. The preferred hand was strapped down by means of elasticised Velcro straps which were attached to each end to ensure that the thumb and little finger stayed on the apparatus. The other hand was assigned to a remote spot on the table.

The noxious stimulus was a white-noise tone of $87 \mathrm{db}$ delivered from a $15 \mathrm{~cm}$ speaker located in the ceiling directly over the subject's head.

Subjects were seated at a table and observed through a one-way mirror from an adjacent room where control and recording equipment were housed.

\section{Procedure}

Subjects were seated in front of the shuttlebox and their preferred hand was strapped to the apparatus. They were instructed: "All I can tell you is that you are going to hear a loud, unpleasant noise that sounds like radio static. There is something you can do about it. Your job is to keep it off as much as possible." (In the Bleiberg, et al study ${ }^{11}$ this last sentence was not included.) The experimenter then left the room and initiated the computer-controlled programme.

Each subject was run until she/he reached a criterion of eight avoidances out of a block of 10 consecutive possible avoidances. A trial was initiated with the onset of the light (CS) on the side to be shuttled from. If no response (shift to the alternate side) occurred within 5 seconds, the $87 \mathrm{db}$ white noise (UCS) was presented. A response within 5 seconds of CS onset precluded any noise and terminated the CS (avoidance). A response after 5 seconds terminated both $C S$ and UCS (escape). Within each block of five avoidance-possible trials there was one additional trial (randomly distributed) which was designated as an escape-only trial. On these trials the light and noise came on simultaneously, and the subject 
only could escape. A spontaneous crossover, that is, responding during the intertrial interval, resulted in UCS presentation which was terminated by returning to the original side. The intertrial interval was 5 seconds.

If the subject failed to reach criterion before 40 avoidance trials were run, she or he received up to an additional $\mathbf{4 0}$ more trials (plus eight escape-only trials) to reach criterion. A randomly assigned half of these subjects had the overhead light turned off during these additional trials, making the CS more salient. The only illumination other than CS onset was a $40 \mathrm{~W}$ desk lamp resting on the floor behind the subject, providing dim ambient lighting. All subjects were forewarned of the possibility that the light conditions might be altered during the experiment.

\section{Results}

For the aggregate sample (including CHI, CVA, and OTH patients), the mean number of trials to criterion was $36 \cdot 8$, with a standard deviation (SD) of $32 \cdot 3$. The mean number of spontaneous crossovers made during these trials, adjusted for opportunities, was 0.29 (SD $=0.27$ ). Mean latency on escape-only trials was $1.4 \mathrm{~s}$ $(\mathrm{SD}=0 \cdot 8)$. Of the 48 total number of subjects run, 12 failed to reach criterion within the initial set of 48 trials (40 avoidance plus eight escape-only trials). Of these, 11 subjects still had not reached criterion within the remaining set of 48 trials. Only one of the six subjects in the warning signal enhancement condition (overhead lights out) learned within the second set of trials.

For the sample broken down into CVA and $\mathrm{CHI}$ groups, the data for mean number of trials to criterion, mean number of spontaneous crossovers (adjusted for relative number of pre-criterion trials run) and mean latencies on escape-only trials are presented in table 1.

The $\mathrm{CHI}$ group required significantly more trials to reach the avoidance criterion than did the CVA group $(\mathrm{t}(26)=3.69, \mathrm{p}<0.001)$. The $\mathrm{CHI}$ group also made more spontaneous crossovers (adjusted) than did the CVA group ( $t(26)=1.69)$, but the difference fell just short of the 0.05 level. Despite the relative superiority of the CVA group on avoidance behaviour, there were no significant differences between the two groups in escape-only latencies.

Table 1 Mean responding on three response parameters for Closed Head Injured (CHI) and Cerebrovascular Accident (CVA) groups

\begin{tabular}{llll}
\hline $\begin{array}{l}\text { Trials to } \\
\text { criterion }\end{array}$ & $\begin{array}{l}\text { Spont. } \\
\text { cross. }\end{array}$ & $\begin{array}{l}\text { Escape } \\
\text { latencies } \\
\text { (seconds) }\end{array}$ \\
\hline CHI & $42 \cdot 1$ & 0.34 & 1.27 \\
CVA & $14 \cdot 1$ & 0.13 & $1 \cdot 19$ \\
\hline
\end{tabular}

In order to determine whether the differences in avoidance performance were due to differences in cognitive or psychomotor ability between the CHI and CVA groups, performance on the individual tests of the Halstead-Reitan Neuropsychological Test Battery was compared. As is seen in table 2, of the 13 test variables, 11 showed no differences between groups. Of the two significant differences, Performance IQ and Tactual Performance Test-Memory, both were higher in the $\mathrm{CHI}$ group. Thus, the $\mathrm{CHI}$ group had equivalent or better cognitive and psychomotor ability compared to the CVA group, but performed worse than the CVA group on the avoidance measures. Mean age showed no significant difference between groups.

The distribution of non-learners in the three groups was CHI (8 out of 21), CVA ( 0 out of 7) and OTH (4 out of 20). If the avoidance scores of the eight nonlearners are removed from the $\mathrm{CHI}$ data, the remaining 13 subjects show a mean trials to criterion score of 18.9 which is not significantly different from the CVA subjects' mean of $14.1(\mathrm{t}(18)=1.45$, n.s.).

\section{Discussion}

The present data replicate and extend the findings of Bleiberg, et al. ${ }^{11} \mathrm{CHI}$ patients showed significantly greater anticipatory behaviour deficit than did CVA patients. This was the case even though the $\mathrm{CHI}$ and CVA patients had equivalent escape learning ability. Moreover, the CHI group's performance on the Wechsler scales and on the individual tests of the Halstead-Reitan Battery was equivalent to or better than the CVA group's performance, indicating that the anticipatory behaviour deficit in the CHI group was not simply a function of generalised cognitive

Table 2 Mean age and test scores for $C V A$ and $C H I$ groups

\begin{tabular}{|c|c|c|}
\hline Variable & $C V A$ & $\mathrm{CHI}$ \\
\hline Full Scale IQ & $80 \cdot 3$ & $89 \cdot 7$ \\
\hline Verbal IQ & $83 \cdot 3$ & 91.6 \\
\hline Performance IQ & $78 \cdot 3$ & $88 \cdot 0^{*}$ \\
\hline Weschsler Memory Quotient & $87 \cdot 7$ & $97 \cdot 5$ \\
\hline \multicolumn{3}{|l|}{ Tactual Performance Test } \\
\hline Total Time (Minutes) & $20 \cdot 9$ & $18 \cdot 8$ \\
\hline $\begin{array}{l}\text { Memory } \\
\text { Location }\end{array}$ & $5 \cdot 0$ & $6 \cdot 8^{*}$ \\
\hline Location & 1.9 & \\
\hline Category Test (Errors) & $52 \cdot 3$ & $50 \cdot 7$ \\
\hline \multicolumn{3}{|l|}{ Trailmaking Test } \\
\hline $\begin{array}{l}\text { Part A (Seconds) } \\
\text { Part B (Seconds) }\end{array}$ & $\begin{array}{r}64 \cdot 6 \\
231.9\end{array}$ & $\begin{array}{r}48.5 \\
141.9\end{array}$ \\
\hline \multicolumn{3}{|l|}{ Seashore Rhythm Test } \\
\hline \\
\hline (Errors) & $17 \cdot 3$ & $10 \cdot 2$ \\
\hline Finger Tapping Speed & $28 \cdot 7$ & $42 \cdot 9$ \\
\hline Age (yr) & $40 \cdot 7$ & $33 \cdot 3$ \\
\hline
\end{tabular}


impairment. CHI patients thus showed disproportionate anticipatory behaviour deficit in relation to escape ability and to cognitive ability in comparison to CVA patients.

The present investigation modified the methodology of the Bleiberg, et al study ${ }^{11}$ by providing subjects with more explicit instructions to avoid the aversive stimulus, and by shortening the experimental procedure through the use of a trials-to-criterion cutoff. The use of instructions which established a clear expectation that avoidance responses were the desired behaviour did not alter the magnitude of anticipatory behaviour deficit in CHI patients. Consequently, this dispels the possibility that the anticipatory behaviour deficit demonstrated in the prior study might have been due to the inability of certain patients to discern the response demands of the situation.

Another alternative hypothesis explored in the current study was that anticipatory behaviour deficit is a manifestation of slow learning rather than a specific anticipatory deficit. Since 11 out of 12 subjects who did not reach criterion in the initial 48 trials had not learned after an additional 48 opportunities, this hypothesis can be rejected. Also, the failure to find improved performance from non-learning subjects who received enhanced CS presentations (overhead lights turned off) suggests that the anticipatory behaviour deficit phenomenon is not attributable to lack of cue saliency. Anticipatory behaviour deficit thus appears to be a relatively dense deficit. It is a robust phenomenon that is not affected by changes in the way it is measured. Most subjects either are "fast learners" or "non-learners," with few subjects being "slow learners."

The distribution of non-learners, with most in the $\mathrm{CHI}$ diagnostic category, some in the OTH but none in CVA, indicates that anticipatory behaviour deficit is a more probable but not an exclusive debility of closed head injured patients. None of the measures used in this study identified the non-learning subjects as distinct from other subjects.

Given the replicability of the anticipatory behaviour deficit phenomenon and the development of an efficient and brief (4-7 $\mathrm{min}$ ) procedure to measure it, the question arises as to the practical or clinical implications of anticipatory behaviour deficit: what does the presence or absence of anticipatory behaviour deficit imply regarding a patient's functioning and adequacy? A "face validity" approach to the question is all that is possible with the present data. These results may suggest that patients with anticipatory behaviour deficit will show deficits in situations requiring that current behaviour be regulated on the basis of expected future consequences, and that in CHI patients this deficit will be disproportionately severe in comparison to neuropsychological test performance on the Halstead-Reitan Battery and the Wechsler scales. External validation studies are needed to assess the accuracy of this face validity interpretation and to examine the relation between anticipatory behaviour deficit and behavioural outcome in closed head injured patients.

\section{References}

1 Luria AR. Frontal lobe syndromes. In: Vinken PV, Bruyn GW, eds. Handbook of Clinical Neurology, Vol. 2, 725-57. New York: Elsevier-North Holland Publishing Co., 1969.

2 Luria AR, Hornskaya ED. Disturbances in the regulative role of speech with frontal lobe lesions. In: Warren JM, Akert K, eds. The Frontal Granular Cortex and Behavior. New York: McGraw, 1964.

3 Denny-Brown D. The frontal lobes and their functions. In: Feiling A, ed. Modern Trends in Neurology, 13-58. New York: Hoeber-Harper, 1951.

4 Oppenheimer H. Clinical Psychiatry: Issues and Challenges. New York: Harper \& Row, 1971.

5 Nauta WJH. The problem of the frontal lobe. J Psychiatric Res 1971;8:167-87.

6 Butter CM, Snyder DR. Alterations in aversive and aggressive behaviors following orbital frontal lesions in rhesus monkeys. Acta Neurobiol Exp 1972;32:525-65.

7 Tanaka D. Effects of selective prefrontal decortication on escape behavior in the monkey. Brain Res 1973;53:161-73.

8 Waterhouse IK. Effects of prefrontal lobotomy on conditioned fear and food responses in monkeys. J Comp 'Physiol Psychol 1957;50:81-8.

9 Fuster JM. The Prefrontal Cortex: Anatomy, Physiology, and Neuropsychology of the Frontal Lobe. New York: Raven Press, 1980.

10 Tanaka D. Sparing of an escape response following serial prefrontal decortication in the monkey. Brain Res 1974;54:195-201.

11 Bleiberg J, Freedman PE, Schueneman AL, Merbitz C, Swartz J. Anticipatory behavior deficits following brain injury. Int J Clin Neuropsychol 1985;7:153-6.

12 Grant DA. Classical and operant conditioning. In: Melton AW, ed. Categories of Human Learning. New York: Academic Press, 1964. 